DOI: $10.2478 /$ auom-2014-0033

\title{
On the torsion group of elliptic curves induced by $D(4)$-triples
}

\author{
Andrej Dujella and Miljen Mikić
}

\begin{abstract}
A $D(4)$ - $m$-tuple is a set of $m$ integers such that the product of any two of them increased by 4 is a perfect square. A problem of extendibility of $D(4)-m$-tuples is closely connected with the properties of elliptic curves associated with them. In this paper we prove that the torsion group of an elliptic curve associated with a $D(4)$-triple can be either $\mathbb{Z} / 2 \mathbb{Z} \times \mathbb{Z} / 2 \mathbb{Z}$ or $\mathbb{Z} / 2 \mathbb{Z} \times \mathbb{Z} / 6 \mathbb{Z}$, except for the $D(4)$-triple $\{-1,3,4\}$ when the torsion group is $\mathbb{Z} / 2 \mathbb{Z} \times \mathbb{Z} / 4 \mathbb{Z}$.
\end{abstract}

\section{Introduction}

Let $n$ be a given nonzero integer. A set of $m$ nonzero integers $\left\{a_{1}, a_{2}, \ldots, a_{m}\right\}$ is called a $D(n)$-m-tuple (or a Diophantine $m$-tuple with the property $D(n)$ ) if $a_{i} a_{j}+n$ is a perfect square for all $1 \leq i<j \leq m$. Diophantus found the $D(256)$-quadruple $\{1,33,68,105\}$, while the first $D(1)$-quadruple, the set $\{1,3,8,120\}$, was found by Fermat (see [1], [2]).

One of the most interesting questions in the study of $D(n)$-m-tuples is how large these sets can be. In this paper we will examine sets with the property $D(4)$. Mohanty and Ramasamy [17] were first to achieve a significant result on the nonextendibility of $D(4)$ - $m$-tuples. They proved that a $D(4)$ quadruple $\{1,5,12,96\}$ cannot be extended to a $D(4)$-quintuple. Kedlaya [14]

Key Words: $D(4)$-triples, elliptic curves, torsion group.

2010 Mathematics Subject Classification: Primary 11G05.

Received: April, 2013.

Revised: June, 2013

Accepted: June, 2013 
later proved that if $\{1,5,12, d\}$ is a $D(4)$-quadruple, then $d$ has to be 96 . Dujella and Ramasamy [9] generalized this result to the parametric family of $D(4)$-quadruples $\left\{F_{2 k}, 5 F_{2 k}, 4 F_{2 k+2}, 4 L_{2 k} F_{4 k+2}\right\}$ involving Fibonacci and Lucas numbers. Other generalization to a two-parametric family of $D(4)$-triples can be found in [13]. Dujella [6] proved that there does not exist a $D(1)$ sextuple and that there are only finitely many $D(1)$-quintuples. By observing congruences modulo 8 , it is not hard to conclude that a $D(4)$ - $m$-tuple can contain at most two odd numbers (see [9, Lemma 1]). Thus, the results from [6] imply that there does not exist a $D(4)-8$-tuple and that there are only finitely many $D(4)-7$-tuples. Filipin $[10,11]$ significantly improved these results by proving that there does not exist a $D(4)$-sextuple and that there are only finitely many $D(4)$-quintuples.

Let $\{a, b, c\}$ be a $D(4)$-triple. Then there exist nonnegative integers $r, s, t$ such that

$$
a b+4=r^{2}, a c+4=s^{2}, b c+4=t^{2} .
$$

In order to extend this triple to a quadruple, we have to solve the system

$$
a x+4=\square, \quad b x+4=\square, \quad c x+4=\square .
$$

We assign to the system (2) the elliptic curve

$$
E: y^{2}=(a x+4)(b x+4)(c x+4) .
$$

The purpose of this paper is to examine possible forms of torsion groups of elliptic curves obtained in this manner. Additional motivation for this paper is a gap found in the proof of [4, Lemma 1] concerning torsion groups of elliptic curves induced by $D(1)$-triples. Namely, if $\left\{a^{\prime}, b^{\prime}, c^{\prime}\right\}$ is a $D(1)$-triple, then $\left\{2 a^{\prime}, 2 b^{\prime}, 2 c^{\prime}\right\}$ is a $D(4)$-triple. Thus, the proof of Lemma 2 in present paper also provides a valid proof of [4, Lemma 1].

\section{Torsion group of $E$}

The coordinate transformation

$$
x \mapsto \frac{x}{a b c}, y \mapsto \frac{y}{a b c}
$$

applied on the curve E leads to the elliptic curve

$$
E^{\prime}: y^{2}=(x+4 b c)(x+4 a c)(x+4 a b) .
$$

There are three rational points on $E^{\prime}$ of order 2 :

$$
A^{\prime}=(-4 b c, 0), B^{\prime}=(-4 a c, 0), C^{\prime}=(-4 a b, 0),
$$


and also other obvious rational points

$$
P^{\prime}=(0,8 a b c), S^{\prime}=(16,8 r s t) \text {. }
$$

It is not so obvious, but it is easy to verify that $S^{\prime} \in 2 E^{\prime}(\mathbb{Q})$. Namely, $S^{\prime}=2 R^{\prime}$, where

$$
R^{\prime}=(4 r s+4 r t+4 s t+16,8(r+s)(r+t)(s+t)) .
$$

In this section we will first examine one special case and after that we may assume without the loss of generality that $a, b, c$ are positive integers such that $a<b<c$. Since $\{-a,-b,-c\}$ induces the same curve as $\{a, b, c\}$, a problem may arise only when there are mixed signs. It is easily seen that the only such possible $D(4)$-triple is $\{-1,3,4\}$ (and the equivalent one $\{-4,-3,1\}$ ). The elliptic curve associated with this $D(4)$-triple has rank 0 and the torsion group isomorphic to $\mathbb{Z} / 2 \mathbb{Z} \times \mathbb{Z} / 4 \mathbb{Z}$. In this special case $B^{\prime} \in 2 E^{\prime}(\mathbb{Q})$, more precisely $B^{\prime}=2 P^{\prime}$, so the point $P^{\prime}$ is of order 4 . Note that in this case the point $R^{\prime}$ is also of order 4 since $R^{\prime}=P^{\prime}+A^{\prime}$ and thus $2 R^{\prime}=2 P^{\prime}$.

Thus, we assume from now on that $a, b, c$ are positive integers such that $a<b<c$.

Lemma 1. If $\{a, b, c\}$ is $D(4)$-triple, then $c=a+b+2 r$ or $c>a b+a+b+1>$ $a b$.

Proof. By [5, Lemma 3], there exists an integer

$$
e=4(a+b+c)+2(a b c-r s t)
$$

and nonnegative integers $x, y, z$ such that

$$
\begin{aligned}
& a e+16=x^{2}, \\
& b e+16=y^{2}, \\
& c e+16=z^{2}
\end{aligned}
$$

and $c=a+b+\frac{e}{4}+\frac{1}{8}(a b e+r x y)$. From (7), it follows that $e \geq 0$ (the case $e=-1$ implies $c \leq 16$, but the only such $D(4)$-triple $\{1,5,12\}$ does not satisfy (5) and (6)). For $e=0$ we get $c=a+b+2 r$, while for $e \geq 1$ we have $c>\frac{1}{4} a b e+a+b+\frac{e}{4}$. By observing congruences modulo 8 , we can easily prove that at most two of the integers $a, b, c$ are odd, which implies that $a b c-r s t$ is even. Hence, from $(4)$ we conclude that $e \equiv 0(\bmod 4)$. It follows $e \geq 4$ and thus $c>a b+a+b+1$. 
Remark 1. Filipin (see [12, Lemma 4]) proved that $c=a+b+2 r$ or $c>\frac{1}{4} a b e$. Lemma 1 may be considered as a slight improvement of that result.

Remark 2. Lemma 1 implies $c \geq a+b+2 r$. Indeed, the inequality $a b+a+b+1 \geq$ $a+b+2 r$ is equivalent to $(r-3)(r+1) \geq 0$, and this is satisfied for all $D(4)$ triples with positive elements.

Remark 3 . The statement of Lemma 1 is sharp the in sense that the inequality $c>a b$ cannot be replaced by $c>(1+\varepsilon) a b$ for any fixed $\varepsilon>0$. Indeed, for an integer $k \geq 3$, if we put $a=k^{2}-4, b=k^{2}+2 k-3, c=k^{4}+2 k^{3}-3 k^{2}-4 k$, then $\{a, b, c\}$ is a $D(4)$-triple and $\lim _{k \rightarrow \infty} \frac{c}{a b}=1$.

In the next lemma we show that $E^{\prime}$ cannot have a point of order 4 . We follow the strategy of the proof of an analogous result for $D(1)$-triples [4, Lemma 1]. However, we have noted a serious gap in the proof of $[4$, Lemma 1]. Namely, [4, formula (7)] should be $\left(\beta^{2}-1\right)^{2}=b\left(4 c \beta^{2}-a^{2} b-2 a\left(1+\beta^{2}\right)\right)$, instead of $\left(\beta^{2}-1\right)^{2}=b\left(4 c-a^{2} b-2 a\left(1+\beta^{2}\right)\right)$, so later arguments are not accurate in the case $\beta \neq 1$. Here we will prove more general result, but by taking $a, b, c$ to be even, in the same time we fill the mentioned gap in the proof of $[4$, Lemma 1].

Lemma 2. $A^{\prime}, B^{\prime}, C^{\prime} \notin 2 E^{\prime}(\mathbb{Q})$

Proof. If $A^{\prime} \in 2 E^{\prime}(\mathbb{Q})$, then the 2-descent Proposition [15, 4.2, p.85] implies that $c(a-b)$ is a square. But $c(a-b)<0$, a contradiction. Similarly, $B^{\prime} \notin$ $2 E^{\prime}(\mathbb{Q})$. If $C^{\prime} \in 2 E^{\prime}(\mathbb{Q})$, then

$$
\begin{aligned}
& a(c-b)=X^{2}, \\
& b(c-a)=Y^{2}
\end{aligned}
$$

for integers $X$ and $Y$.

If $\{a, b, c\}$ is a $D(4)$-triple where $a<b<c$, then $c=a+b+2 r$ or $c>a b+a+b+1$ by Lemma 1 .

Assume first that $c=a+b+2 r$. From (8) and (9), we get that $a=k x^{2}$, $c-b=k y^{2}, b=l z^{2}, c-a=l u^{2}$, where $k, l, x, y, z, u$ are positive integers. We have $c=k x^{2}+l u^{2}=k y^{2}+l z^{2}$, and from $c=a+b+2 r$ we get

$$
2 r=k\left(y^{2}-x^{2}\right)=l\left(u^{2}-z^{2}\right) .
$$

By squaring (10), we obtain

$$
4 r^{2}=16+4 a b=16+4 k l x^{2} z^{2}=k^{2}\left(y^{2}-x^{2}\right)^{2}=l^{2}\left(u^{2}-z^{2}\right)^{2},
$$


which implies that $k \in\{1,2,4\}$ and $l \in\{1,2,4\}$. Since $k l$ is not a perfect square (otherwise $(2 r)^{2}=16+(2 x z \sqrt{k l})^{2}$ which implies $2 r=5$ ), we may take without loss of generality $k=1, l=2$ or $k=2, l=4$. For $k=1$, $l=2$, we have $4 r^{2}=16+8 x^{2} z^{2}$, which implies $r^{2}=4+2 x^{2} z^{2}$, which leads to the conclusion that $r$ is even and $x z$ is even. Therefore, $r^{2} \equiv 4(\bmod 8)$ and $r \equiv 2(\bmod 4)$. But from $2 r=2\left(u^{2}-z^{2}\right)$ we conclude $u^{2}-z^{2} \equiv 2$ $(\bmod 4)$, and that is impossible. If $k=2, l=4$, then $4 r^{2}=16+32 x^{2} z^{2}$, which implies $r^{2}=4+8 x^{2} z^{2}$, thus $r^{2} \equiv 4(\bmod 8)$ and $r \equiv 2(\bmod 4)$. But from $2 r=2\left(y^{2}-x^{2}\right)$ we conclude $y^{2}-x^{2} \equiv 2(\bmod 4)$, and that is impossible.

Assume now that $c>a b+a+b+1>a b$.

Let us write the conditions (8) and (9) in the form

$$
\begin{aligned}
& a c-a b=s^{2}-r^{2}=(s-\alpha)^{2}, \\
& b c-a b=t^{2}-r^{2}=(t-\beta)^{2},
\end{aligned}
$$

where $0<\alpha<s, 0<\beta<t$. Then we have

$$
r^{2}=2 s \alpha-\alpha^{2}=2 t \beta-\beta^{2} .
$$

From (13) we get

$$
4(b c+4) \beta^{2}=\left(a b+4+\beta^{2}\right)^{2}
$$

and

$$
\left(\beta^{2}-4\right)^{2}=b\left(4 c \beta^{2}-a^{2} b-2 a\left(4+\beta^{2}\right)\right) .
$$

From (14) we conclude that either $\beta=1$ or $\beta=2$ or $\beta^{2} \geq \sqrt{b}+4$.

If $\beta=1$, then

$$
b\left(4 c-a^{2} b-10 a\right)=9
$$

which implies $b \mid 9$, but that is possible only for $b=9$ (there are no $D(4)$-triples with $b<4)$. This implies $a=5$, but (15) then gives $c=69$ and $\{5,9,69\}$ is not a $D(4)$-triple.

If $\beta=2$, then from (14) we find that

$$
c=\frac{a^{2} b+16 a}{16} .
$$

Now we have

$$
s^{2}=a c+4=\frac{1}{16}\left(a^{3} b+16 a^{2}+64\right)=\frac{1}{16}\left(a^{2} r^{2}+12 a^{2}+64\right) .
$$

Hence $s^{2}>\left(\frac{a r}{4}\right)^{2}$ and $s^{2}<\left(\frac{a r+8}{4}\right)^{2}$. Therefore we have to consider several cases: 
1. $s^{2}=\left(\frac{a r+n}{4}\right)^{2}$, where $n$ is odd. That is equivalent to

$$
2 a(r n-6 a)=64-n^{2} .
$$

The left hand side of (17) is even and the right hand side is odd, a contradiction.

2. $s^{2}=\left(\frac{a r+2}{4}\right)^{2}$, or equivalently $a(r-3 a)=15$. The cases $a \leq 3$ and (16) imply that $c<b$. The case $a=5$ gives the triple $\{5,64,105\}$ that does not satisfy $c>a b$ ( $c$ equals $a+b+2 r$ ), and $a=15$ leads to $15 b+4=46^{2}$ which has no integer solutions.

3. $s^{2}=\left(\frac{a r+4}{4}\right)^{2}$, or equivalently $a(2 r-3 a)=12$. We conclude that $a$ must be even and we get triples: $\{2,16,6\}$ (with $c<b$ ) and $\{6,16,42\}$ (with $c=a+b+2 r)$, so we can eliminate this case.

4. $s^{2}=\left(\frac{a r+6}{4}\right)^{2}$ is equivalent to $3 a(r-a)=7$, which is clearly impossible.

Thus, we may assume that $\beta^{2} \geq \sqrt{b}+4$, which implies

$$
\beta>\max \{\sqrt[4]{b}, 2\}
$$

The function $f(\beta)=t^{2}-(t-\beta)^{2}$ is increasing for $0<\beta<t$. Thus we have

$$
a b=t^{2}-(t-\beta)^{2}-4>2 t \sqrt[4]{b}-\sqrt{b}-4>2 \sqrt{b c} \sqrt[4]{b}-\sqrt{b}-4,
$$

which implies $a b>\sqrt{b c} \sqrt[4]{b}$, because $\sqrt{b}(\sqrt{c} \sqrt[4]{b}-1)>4$ (since $b \geq 4$ and $c \geq 12$, which follows from the fact that $\{3,4,15\}$ and $\{1,5,12\}$ are $D(4)$-triples with smallest $b$ and $c$ respectively). This further gives

$$
c<a^{2} \sqrt{b}
$$

We will use (4) to define the integer $d_{-}$as

$$
d_{-}=\frac{e}{4}=a+b+c+\frac{a b c-r s t}{2}
$$

Then $d_{-} \neq 0$ (since $\left.c \neq a+b+2 r\right)$ and $\left\{a, b, c, d_{-}\right\}$is a $D(4)$-quadruple. In particular,

$$
a d_{-}+4=\left(\frac{r s-a t}{2}\right)^{2} \text {. }
$$

Moreover,

$$
c=a+b+d_{-}+\frac{1}{2}\left(a b d_{-}+\sqrt{(a b+4)\left(a d_{-}+4\right)\left(b d_{-}+4\right)}\right)>a b d_{-}
$$


(see the proof of Lemma 1). By comparing this with (19), we get

$$
d_{-}<\frac{a}{\sqrt{b}}
$$

Therefore, we have $d_{-}<a<b$ which implies that $b$ is the largest element in the $D(4)$-triple $\left\{a, b, d_{-}\right\}$. Thus, by Remark $2, b \geq a+d_{-}+2 \sqrt{a d_{-}+4}$ or equivalently $d_{-} \leq a+b-2 r$. Let us define also

$$
c^{\prime}=a+b+d_{-}+\frac{1}{2}\left(a b d_{-}-\sqrt{(a b+4)\left(a d_{-}+4\right)\left(b d_{-}+4\right)}\right) .
$$

We have

$$
\begin{aligned}
c c^{\prime} & =\left(a+b+d_{-}+\frac{1}{2} a b d_{-}\right)^{2}-\frac{1}{4}(a b+4)\left(a d_{-}+4\right)\left(b d_{-}+4\right) \\
& =\left(a+b+d_{-}\right)^{2}-4 a b-4 a d_{-}-4 b d_{-}-16 \\
& =\left(a+b-d_{-}\right)^{2}-4 r^{2}=\left(a+b+2 r-d_{-}\right)\left(a+b-2 r-d_{-}\right) \geq 0 .
\end{aligned}
$$

This implies

$$
c<2\left(a+b+d_{-}+\frac{1}{2} a b d_{-}\right)<4 b+a b d_{-}<2 a b d_{-} .
$$

(we use here $a d_{-}>4$ which is true because $\left\{a, d_{-}\right\}$is a $D(4)$-pair). Let us denote $p=\frac{r s-a t}{2}$. Then $p>0$ and, by (20), we have $a d_{-}+4=p^{2}$. In order to estimate the size of $p$, we also define $p^{\prime}=\frac{r s+a t}{2}$. Then

$$
p p^{\prime}=\frac{1}{4}\left(a^{2} b c+4 a c+4 a b+16-a^{2} b c-4 a^{2}\right)=a(b+c-a)+4,
$$

and

$$
\begin{gathered}
p<\frac{2 a(c+b)}{2 a t}<\frac{c+b}{\sqrt{b c}}=\frac{\sqrt{c}}{\sqrt{b}}+\frac{\sqrt{b}}{\sqrt{c}}, \\
p>\frac{2(a c+4)}{2 r s}=\frac{s}{r} .
\end{gathered}
$$

Furthermore, we have

$$
\frac{\sqrt{c}}{\sqrt{b}}-\frac{s}{r}=\frac{r \sqrt{c}-s \sqrt{b}}{r \sqrt{b}}=\frac{4 c-4 b}{r \sqrt{b}(r \sqrt{c}+s \sqrt{b})}<\frac{4 c}{2 r s b}<\frac{2 \sqrt{c}}{a b \sqrt{b}},
$$

and thus

$$
p>\frac{\sqrt{c}}{\sqrt{b}}-\frac{2 \sqrt{c}}{a b \sqrt{b}} .
$$


The inequality (19) implies that $c<\frac{a b^{2}}{2}$, and this is equivalent to

$$
\frac{\sqrt{b}}{\sqrt{c}}>\frac{2 \sqrt{c}}{a b \sqrt{b}}
$$

which gives

$$
p>\frac{\sqrt{c}}{\sqrt{b}}-\frac{\sqrt{b}}{\sqrt{c}}
$$

By comparing both estimates for $p$, we get

$$
\left|p-\frac{\sqrt{c}}{\sqrt{b}}\right|<\frac{\sqrt{b}}{\sqrt{c}}
$$

Let us now define an integer $\alpha$ by

$$
2 d_{-} \beta=p+\alpha .
$$

Assume that $\alpha=0$. Then (20) implies that $d_{-}\left(4 \beta^{2} d_{-}-a\right)=4$, thus $d_{-} \in\{1,2,4\}$. We have three cases:

1. $d_{-}=1$, which implies $2 \beta=p$. With this assumption, (12) gives

$$
r^{2}+\frac{p^{2}}{4}=t p
$$

while $c$ satisfies the inequalities

$$
a b<a b+a+b+1<c<a b+2 a+2 b+2<a b+4 b<2 a b
$$

(see Lemma 1 and (23) with $d_{-}=1$ ). The left hand side of (27) is

$$
<a b+4+\frac{c^{2}+2 b c+b^{2}}{4 b c}<a b+4+\frac{a}{4}+1+\frac{1}{2}+\frac{1}{4 a}<a b+\frac{a}{4}+6 .
$$

On the other hand, by (24), the right hand side of (27) is

$>\sqrt{b c}\left(\frac{\sqrt{c}}{\sqrt{b}}-\frac{2 \sqrt{c}}{a b \sqrt{b}}\right)=c-\frac{2 c}{a b}>a b+a+b+1-4=a b+a+b-3$.

By comparing these two estimates for (27), we get

$$
b+\frac{3}{4} a<9
$$

but this is in contradiction with $b \geq 12$ ( $b$ is the largest element in the $D(4)$-triple $\left.\left\{d_{-}, a, b\right\}\right)$.

We treat similarly the other two cases. 
2. $d_{-}=2$, which implies $4 \beta=p$, and this leads to

$$
\frac{b}{2}+\frac{3}{8} a<8,
$$

which is in contradiction with $b \geq 16(D(4)$-triple of the form $\{2, a, b\}$ with the smallest $b$ is $\{2,6,16\})$.

3. $d_{-}=4$ is equivalent to $8 \beta=p$, which leads to

$$
\frac{b}{4}+\frac{3}{16} a<8
$$

but the only $D(4)$-triple of the form $\{4, a, b\}$ with $b<35$ is $\{4,8,24\}$, which does not satisfy (22), so we have a contradiction here as well.

Therefore, we may now assume that $\alpha \neq 0$. We will estimate $2 d_{-} t \beta$ and compare it with $c$. First we will prove

$$
\beta^{2}<\frac{a^{2} b}{c} .
$$

Since $\beta<t$, and the case $\beta=t-1$ gives $b(c-a)=1$, which is impossible, we conclude that $t \geq \beta+2$. This implies $t \beta \geq \beta^{2}+2 \beta$, and $a b-t \beta \geq 2 \beta-4>0$ because of (18). Hence, we get $t \beta<a b$, and this clearly implies (28).

Therefore,

$$
0<d_{-} \beta^{2}<\frac{d_{-} a^{2} b}{c}<a .
$$

From $2 t \beta=r^{2}+\beta^{2}>a b+4$, we get $2 d_{-} t \beta>a b d_{-}+4 d_{-}$. On the other hand,

$$
d_{-} \beta^{2}<\frac{d_{-} a^{2} b}{c} \Leftrightarrow 2 d_{-} t \beta<a b d_{-}+4 d_{-}+\frac{d_{-} a^{2} b}{c}<a b d_{-}+4 d_{-}+a .
$$

By combining these two estimates, we get

$$
a b d_{-}+4 d_{-}<2 d_{-} t \beta<a b d_{-}+4 d_{-}+a .
$$

By comparing (29) with (21) and (23), we conclude that

$$
\left|2 d_{-} t \beta-c\right|<4 b .
$$

By combining the estimate (26) for $p$ with the trivial estimate for $\alpha$, namely $|\alpha| \geq 1$, we get

$$
\left|2 d_{-} \beta-\frac{\sqrt{c}}{\sqrt{b}}\right|=\left|p+\alpha-\frac{\sqrt{c}}{\sqrt{b}}\right| \geq 1-\frac{\sqrt{b}}{\sqrt{c}} .
$$


Note that $a d_{-}>26$. Namely, only $D(4)$-pairs such that $a d_{-} \leq 26$ are $\{1,5\},\{1,12\},\{1,21\},\{2,6\},\{3,4\}$ and $\{3,7\}$. From first three pairs, respecting (21) and (22), we find triples

$$
\{5,12,96\},\{12,21,320\},\{12,96,1365\},\{21,32,780\},\{21,320,7392\}
$$

that do not satisfy (8) nor (9). From the last three pairs we cannot obtain a $D(4)$-triple because of (22).

Finally, we obtain

$$
\begin{gathered}
\left|2 d_{-} t \beta-c\right|=\left|2 d_{-} t \beta-t \frac{\sqrt{c}}{\sqrt{b}}+t \frac{\sqrt{c}}{\sqrt{b}}-c\right| \geq t\left|2 d_{-} \beta-\frac{\sqrt{c}}{\sqrt{b}}\right|-\left|t \frac{\sqrt{c}}{\sqrt{b}}-c\right| \\
=t\left|2 d_{-} \beta-\frac{\sqrt{c}}{\sqrt{b}}\right|-\left(t \frac{\sqrt{c}}{\sqrt{b}}-c\right) \geq t\left(1-\frac{\sqrt{b}}{\sqrt{c}}\right)-\left(t \frac{\sqrt{c}}{\sqrt{b}}-c\right) \\
=t\left(1-\frac{\sqrt{b}}{\sqrt{c}}\right)-c\left(\sqrt{1+\frac{4}{b c}}-1\right)>\sqrt{b c}-b-c\left(\sqrt{1+\frac{4}{b c}}-1\right) \\
>\sqrt{a b^{2} d_{-}}-b-\frac{2}{b} \geq b\left(\sqrt{a d_{-}}-1-\frac{1}{72}\right)>4 b
\end{gathered}
$$

which contradicts (30).

Theorem 3. $E^{\prime}(\mathbb{Q})_{\text {tors }} \simeq \mathbb{Z} / 2 \mathbb{Z} \times \mathbb{Z} / 2 \mathbb{Z}$ or $\mathbb{Z} / 2 \mathbb{Z} \times \mathbb{Z} / 6 \mathbb{Z}$.

Proof. By Mazur's theorem [16] which characterizes all possible torsion groups for elliptic curves over $\mathbb{Q}$, since $E^{\prime}$ has three points of order 2 , the only possibilities for $E^{\prime}(\mathbb{Q})_{\text {tors }}$ are $\mathbb{Z} / 2 \mathbb{Z} \times \mathbb{Z} / 2 k \mathbb{Z}$ with $k=1,2,3,4$. But Lemma 2 shows that the cases $k=2,4$ are not possible for an elliptic curve induced by a $D(4)$-triple with positive elements.

Corolary 4. Let $\{a, b, c\}$ be a $D(1)$-triple. Then the torsion group of the elliptic curve $y^{2}=(a x+1)(b x+1)(c x+1)$ is either $\simeq \mathbb{Z} / 2 \mathbb{Z} \times \mathbb{Z} / 2 \mathbb{Z}$ or $\mathbb{Z} / 2 \mathbb{Z} \times \mathbb{Z} / 6 \mathbb{Z}$.

Remark 4. We note that an analogue of Theorem 3 and Corollary 4 is not valid for general $D\left(n^{2}\right)$-triples and their induced elliptic curves

$$
y^{2}=\left(a x+n^{2}\right)\left(b x+n^{2}\right)\left(c x+n^{2}\right) .
$$

For example, for the $D(9)$-triple $\{8,54,104\}$ the torsion group of the induced elliptic curve is $\mathbb{Z} / 2 \mathbb{Z} \times \mathbb{Z} / 4 \mathbb{Z}$. Also, there are examples with torsion group $\mathbb{Z} / 2 \mathbb{Z} \times \mathbb{Z} / 8 \mathbb{Z}$, e.g. for the $D\left(52208405404435206419201940^{2}\right)$-triple

$$
\begin{gathered}
\{3871249317729019929807383,101862056999203416732147408, \\
217448139952121636379025175\}
\end{gathered}
$$

(there are much simpler examples with triples with mixed signs, see e.g. [7]). 
We should also mention that we do not know any example of $D(1)$ or $D(4)$ triples inducing elliptic curves with torsion group $\mathbb{Z} / 2 \mathbb{Z} \times \mathbb{Z} / 6 \mathbb{Z}$. Indeed, it is known that this torsion group cannot appear for certain families of $D(1)$-triples (see $[3,4,8,18])$. Again, there are examples of such curves for general $D\left(n^{2}\right)$ triples. For example, the $D\left(294^{2}\right)$-triple $\{32,539,1215\}$ induces an elliptic curve with torsion group $\mathbb{Z} / 2 \mathbb{Z} \times \mathbb{Z} / 6 \mathbb{Z}$.

\section{References}

[1] L. E. Dickson, A history of the Theory of numbers, Vol. 2, Chelsea, New York, 1966., pp. 513-520.

[2] Diophantus of Alexandria, Arithmetics and the Book of Polygonal Numbers (I. G. Bashmakova, Ed.), Nauka, 1974, (in Russian), pp. 103-104, 232.

[3] A. Dujella, A parametric family of elliptic curves, Acta Arith. 94 (2000), 87-101.

[4] A.Dujella, Diophantine m-tuples and elliptic curves, J. Theor. Nombres Bordeaux 13 (2001), 111-124.

[5] A. Dujella, On the size of Diophantine m-tuples, Math. Proc. Cambridge Philos. Soc. 132 (2002), 23-33.

[6] A. Dujella, There are only finitely many Diophantine quintuples, J. Reine Angew. Math. 566 (2004), 183-214.

[7] A. Dujella, On Mordell-Weil groups of elliptic curves induced by Diophantine triples, Glas. Mat. Ser. III 42 (2007), 3-18.

[8] A. Dujella and A. Pethő, Integer points on a family of elliptic curves, Publ. Math. Debrecen 56 (2000), 321-335.

[9] A. Dujella and A. M. S. Ramasamy, Fibonacci numbers and sets with the property D(4), Simon Stevin 12 (2005), 401-412.

[10] A. Filipin, There does not exist a D(4)-sextuple, J. Number Theory 128 (2008), 1555-1565.

[11] A. Filipin, There are only finitely many D(4)-quintuples, Rocky Mountain J. Math. 41 (2011), 1847-1859.

[12] A. Filipin, An irregular D(4)-quadruple cannot be extended to a quintuple, Acta Arith. 136 (2009), 167-176. 
[13] A. Filipin, Bo He and A. Togbé, On a family of two-parametric D(4)triples, Glas. Mat. Ser. III 47 (2012), 31-51.

[14] K. S. Kedlaya, Solving constrained Pell equations, Math. Comp. 67 (1998), 833-842.

[15] A. Knapp, Elliptic Curves, Princeton Univ. Press, 1992.

[16] B. Mazur, Rational isogenies of prime degree, Invent. Math. 44 (1978), $129-162$.

[17] S. P. Mohanty and A. M. S. Ramasamy, The characteristic number of two simultaneous Pell's equations and its applications, Simon Stevin $\mathbf{5 9}$ (1985), 203-214.

[18] F. Najman, Integer points on two families of elliptic curves, Publ. Math. Debrecen 75 (2009), 401-418.

Andrej DUJELLA,

Department of Mathematics,

University of Zagreb,

Bijenička cesta 30, 10000 Zagreb, Croatia.

Email: duje@math.hr

Miljen MIKIĆ,

Kumičićeva 20, 51000 Rijeka, Croatia

Email: miljen.mikic@gmail.com 